the stool of infected individuals, ${ }^{2}$ this has raised safety concerns for administering FMTs. The US Food and Drug Administration has recommended that only FMT products generated from stools donated before 1 December 2019 should be used until further guidance on SARS-CoV-2 testing protocols are available. ${ }^{3}$ However, the supply of prestored samples has dwindled and the stability of the microbes and the efficacy of FMT after prolonged storage are questionable. ${ }^{4}$ Currently many FMT centres are closed with no available FMT treatment for recurrent Clostridioides difficile Infection ( $\mathrm{rCDI}$ ) or other patients. We aim to share our Health Canada-approved strategy in performing FMTs during the pandemic in our rCDI clinic and our ongoing oncology clinical trials with the hope that more centres can safely restart their FMT programmes. We will focus on the three-stage FMT donor screening pertaining to COVID-19 as other components of the programme remain the same. ${ }^{5}$

Donors are administered a questionnaire, over the phone, before each sample drop-off. The screening questionnaire includes COVID-19 symptoms, whether they have previously tested positive for COVID-19, have travelled outside of Canada in the last 14 days, had close unprotected contact with a confirmed case of COVID-19 or worked in any facility with a declared COVID-19 outbreak. If the answer is yes to any of these questions, then they are excluded and referred for SARS-CoV-2 testing (figure 1). If testing is negative and they become asymptomatic, they are eligible for rescreening after 14 days. If the donors passed the questionnaire, they would visit the clinic and drop off their stool samples. During each visit donors provide a nasopharyngeal swab for COVID-19 testing (reverse transcription Polymerase chain reaction (RT PCR) and a portion of the stool sample is tested for SARS-CoV-2 RT PCR. ${ }^{6}$ Stool

\section{Enhanced donor screening for faecal microbial transplantation during COVID-19}

We read with interest the recent article by Ianiro et $a l^{1}$ which is a guidance document on faecal microbial transplantation (FMT) during the COVID-19 pandemic. This guidance document advocated stool testing for SARS-CoV-2 and nasopharyngeal sampling was recommended. The pandemic has imposed new challenges to healthcare systems around the world. Since SARS-CoV-2 RNA can be found in donations are then processed and quaranable. Donors are advised to quarantine if there is a positive result and the Ontario Public Health is notified and stool samples disposed of following a biological hazard protocol. Stool samples are processed into capsules. ${ }^{7}$ We chose FMT delivery via capsules as the preferred method as it is non-invasive and does not generate aerosols. Enema is administered to patients unable to swallow capsules, with staff wearing gowns, surgical masks, gloves and face shield in a dedicated room. All patients are followed up by telephone for 30 days post-FMT. All donors and 


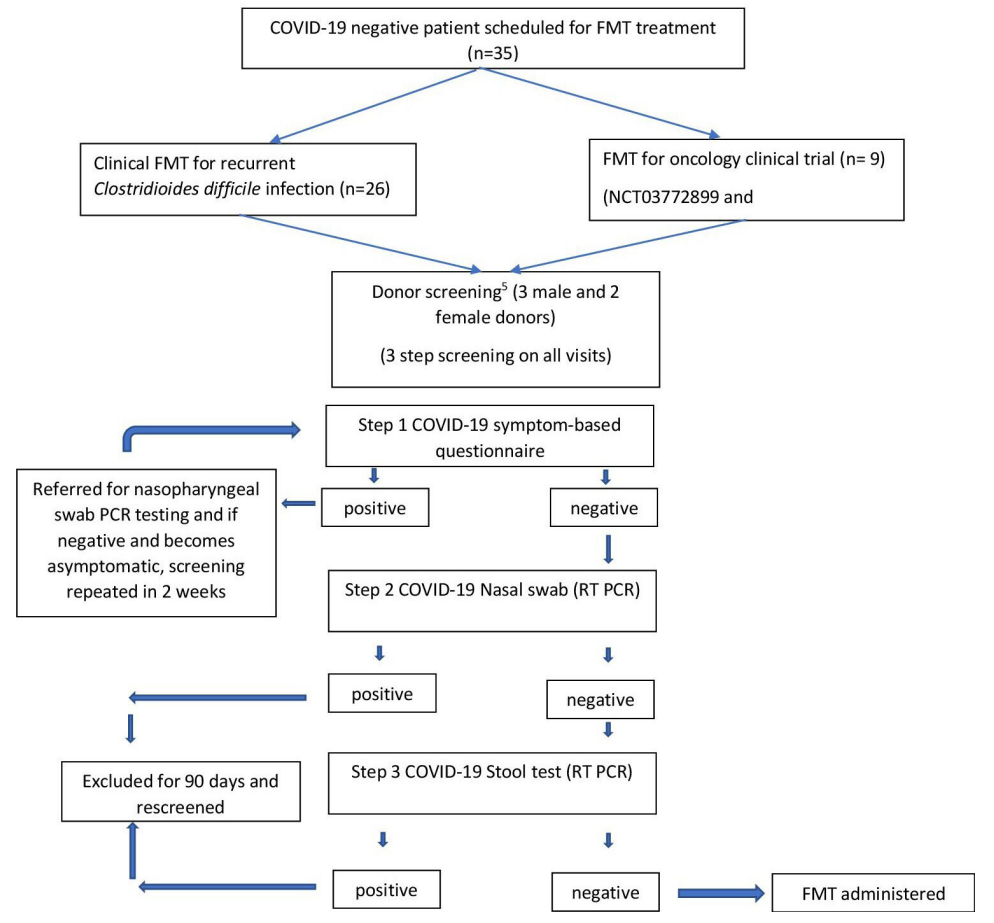

Figure 1 Enhanced FMT donor screening algorithm following the COVID-19 pandemic. FMT, faecal microbial transplantation; RT PCR, reverse transcription Polymerase chain reaction.

recipients provide written informed consent.

We have administered FMTs to 35 patients ( 9 enemas and 26 by capsules) starting August 2020 with no cases of COVID-19 reported in the recipients following FMT (figure 1). No donors tested positive for SARS-CoV-2. No donors or recipients have yet been vaccinated for COVID-19. We will continue the same protocol irrespective of donor or recipient vaccination status. Ianiro et $a l^{8}$ have also published their experience on maintaining the FMT service during the pandemic. ${ }^{8}$

To our knowledge this is the largest cohort of patients undergoing FMT reported since the onset of the pandemic and the first reported to include stool SARS-CoV-2 testing. Our data demonstrate that FMT can be safely performed despite pandemic restrictions.

Seema Nair Parvathy $\oplus^{1,2}$ John G Lenehan ${ }^{3,4}$ Ricardo Fernandes, ${ }^{3,4}$ Susan M Poutanen, ${ }^{5,6}$ Susy Hota, ${ }^{7,8}$ Saman Maleki Vareki, ${ }^{4,9,10}$ Michael Silverman ${ }^{1,2,10}$

${ }^{1}$ Infectious Diseases, St.Joseph's Health Care, London, Ontario, Canada

${ }^{2}$ Infectious Diseases, Medicine, Western University, London, Ontario, Canada

${ }^{3}$ Division of Medical Oncology, Department of

Oncology, Western University, London, Ontario, Canada

${ }^{4}$ Division of Experimental Oncology, Department of

Oncology, Western University, London, Ontario, Canada
${ }^{5}$ Departments of Laboratory Medicine and Pathobiology and Medicine, University of Toronto, Toronto, Ontario, Canada

${ }^{6}$ Department of Microbiology \& Division of Infectious Diseases, University Health Network and Sinai Health, Toronto, Ontario, Canada

${ }^{7}$ Infection Prevention and Control Department, University Health Network, Toronto, Ontario, Canada ${ }^{8}$ Department of Medicine, University of Toronto, Toronto, Ontario, Canada

${ }^{9}$ Departments of Pathology and Laboratory Medicine, Western University, London, Ontario, Canada

${ }^{10}$ Lawson Health Research Institute, London, Ontario, Canada

Correspondence to Dr Michael Silverman, Department of Medicine, Western University, London ON N6A 3K7, Canada; Michael.Silverman@sjhc.Iondon. on.ca

Twitter Saman Maleki Vareki @smimmunology

Contributors SNP and MS designed the study, implemented the study and prepared the manuscript. JGL, RF and SMV contributed to the study design and implementation. SMP and SH helped in designing the Stool Covid SARS-CoV-2 test.

Funding This study was funded by the Lotte and John Hecht Memorial Foundation (grant \#4324).

Competing interests None declared.

Patient and public involvement Patients and/or the public were not involved in the design, or conduct, or reporting, or dissemination plans of this research.

Patient consent for publication Not required.

Ethics approval Study was approved by the Research Ethics Board of Western University.

Provenance and peer review Not commissioned; externally peer reviewed.

\section{(อ \\ OPEN ACCESS}

Open access This is an open access article distributed in accordance with the Creative Commons Attribution Non Commercial (CC BY-NC 4.0) license, which permits others to distribute, remix, adapt, build upon this work non-commercially, and license their derivative works on different terms, provided the original work is properly cited, appropriate credit is given, any changes made indicated, and the use is non-commercial. See: http:// creativecommons.org/licenses/by-nc/4.0/.

(c) Author(s) (or their employer(s)) 2021. Re-use permitted under CC BY-NC. No commercial re-use. See rights and permissions. Published by BMJ.

\section{D) Check for updates}

To cite Parvathy SN, Lenehan JG, Fernandes $R$, et al. Gut 2021:70:2219-2220.

Received 7 March 2021

Revised 14 March 2021

Accepted 16 March 2021

Published Online First 31 March 2021

Gut 2021;70:2219-2220. doi:10.1136/ gutjnl-2021-324593

\section{ORCID iD}

Seema Nair Parvathy http://orcid.org/0000-0001-6738000X

\section{REFERENCES}

1 laniro G, Mullish BH, Kelly CR, et al. Reorganisation of faecal microbiota transplant services during the COVID-19 pandemic. Gut 2020;69:1555-63.

2 Wang X, Zheng J, Guo L, et al. Fecal viral shedding in COVID-19 patients: clinical significance, viral load dynamics and survival analysis. Virus Res 2020;289:198147.

3 FDA. Safety alert regarding use of fecal microbiota for transplantation and additional safety protections pertaining to SARS-CoV-2 and COVID-19, 2020. Available: https://www.fda.gov/vaccines-bloodbiologics/safety-availability-biologics/safety-alertregarding-use-fecal-microbiota-transplantation-andadditional-safety-protections

4 Craven LGG, Reid G, Silverman M. What are the optimal long-term storage conditions for donor samples used in fecal microbiota transplantation? New Orleans, LA: ASM Microbe, 2017

5 Craven U, Nair Parvathy S, Tat-Ko J, et al. Extended Screening Costs Associated With Selecting Donors for Fecal Microbiota Transplantation for Treatment of Metabolic Syndrome-Associated Diseases. Open Forum Infect Dis. Fall 2017:4:0fx243.

6 Ng SC, Chan FKL, Chan PKS. Screening FMT donors during the COVID-19 pandemic: a protocol for stool SARS-CoV-2 viral quantification. Lancet Gastroenterol Hepatol 2020;5:642-3.

7 Kao D, Roach B, Silva M, et al. Effect of oral Capsule- vs Colonoscopy-Delivered fecal microbiota transplantation on recurrent Clostridium difficile infection: a randomized clinical trial. JAMA 2017;318:1985-93.

8 Ianiro G, Bibbò S, Masucci L, et al. Maintaining standard volumes, efficacy and safety, of fecal microbiota transplantation for $C$. difficile infection during the COVID-19 pandemic: a prospective cohort study. Dig Liver Dis 2020;52:1390-5. 